\title{
Análise da capacidade respiratória em um grupo de praticantes de equoterapia
}

\author{
Analysis of respiratory capacity in a \\ group of equine therapy
}

FisiSenectus. Unochapecó Ano 4, n. 2 - Jul/Dez. 2016 p. $38-43$

Letícia Haiduki. leticia_haiduki@hotmail.com

Estagiária do Curso de Fisioterapia. Universidade Regional Integrada do Alto Uruguai e das Missões - URI Erechim.

Márcia Bairros de Castro.mbairros@uricer.edu.br

Fisioterapeuta. Doutora Docente do Departamento de Ciências da Saúde da Universidade Regional Integrada do Alto Uruguai e das Missões - URI Erechim.

Aline Maria Tonatto. aline.tonatto@yahoo.com Fisioterapeuta do Centro de Fortalecer Equoterapia.

Marisa Calgarotto. marisacalgarotto@hotmail.com Psicóloga do Centro de Fortalecer Equoterapia.

Adriana Marcia Petry Santana.santana.adriana7@gmail.com.br Assistente Social do Centro de Fortalecer Equoterapia.

\section{Resumo}

Introdução: A função muscular respiratória pode ser analisada pela força muscular respiratória, capacidade vital, resistência muscular e complacência pulmonar. A hipotonia e hipertonia, característica das crianças portadoras de necessidades especiais, podem causar uma incapacidade de produzir força muscular respiratória, resultando em um padrão respiratório anormal. Uma das formas de intervenção terapêutica é a equoterapia. Objetivos: verificar os efeitos da equoterapia sobre a capacidade respiratória em um grupo de crianças portadoras de necessidades especiais. Materiais e métodos: a avaliação contou com preenchimento de um questionário de identificação, seguida do teste de manovacuometria. As sessões de equoterapia foram iniciadas após a coleta dos dados. Os pacientes receberam atendimento uma vez por semana, em um período de dez semanas, com o tempo de 30 minutos cada sessão, totalizando dez sessões. Após esse período de dez sessões, os participantes foram reavaliados, conforme protocolo da avaliação inicial, para análise dos resultados. Resultados: observou-se que, após as dez sessões de equoterapia, houve melhoria da força muscular dos praticantes tanto para PEMáx como PIMáx; no entanto, mostrando-se com diferenças significativas em PEMáx. Considerações finais: Os resultados obtidos permitem concluir que essa forma de tratamento pode contribuir para capacidade respiratória dos praticantes. Portanto o movimento tridimensional do cavalo trabalha de forma sinergética e os resultados são alcançados de forma conjunta.

\section{Palavras-chave}

Capacidade respiratória; Equoterapia; Necessidades especiais. 


\begin{abstract}
Introduction: Respiratory muscle function can be analyzed by respiratory muscle strength, vital capacity, muscular endurance and pulmonary compliance. Hypotonia and hypertonia, characteristic of children with special needs may cause an inability to produce respiratory muscle strength, resulting in an abnormal respiratory pattern. One of the forms of therapeutic intervention is equine therapy. Objectives: To verify the effects of equine therapy on respiratory capacity in a group of children with special needs. Materials and methods: the evaluation had to fill out an identification questionnaire, followed by the manovacuometry test. Equine therapy sessions were started after data collection. Patients received care once a week, over a period of ten weeks, with the time of 30 minutes each session, totaling ten sessions. After this period of ten sessions the participants were reassessed, according to the protocol of the initial evaluation to analyze the results. Results: it was observed that after the ten sessions of equine therapy there was improvement of the muscular strength of the practitioners for both PEMáx and PIMáx, however showing with significant differences in PEMáx. Final considerations: The results obtained allow us to conclude that this form of treatment can contribute to the respiratory capacity of the practitioners. Therefore the three-dimensional movement of the horse works synergistically and the results are achieved together.
\end{abstract}

\section{Keywords}

Respiratory capacity; Equine therapy; Special needs.

\section{Introdução}

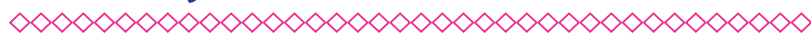

A função muscular respiratória pode ser analisada pela força muscular respiratória, capacidade vital, resistência muscular e complacência pulmonar ${ }^{1}$. Essa força também pode ser mensurada por meio de medidas estáticas, pela Pressão Inspiratória máxima (PIMáx) e pela Pressão Expiratória máxima (PEMáx), e estas podem ser utilizadas para quantificar a força dos músculos respiratório e também para avaliar a resposta ao treinamento muscular respiratório ${ }^{1,2}$.

Nas doenças neuromusculares, as disfunções dos músculos respiratórios induzem o início da insuficiência respiratória, ocorrendo um funcionamento inadequado dos centros nervosos reguladores da respiração ou da atividade muscular, resultando em uma dificuldade da expansão pulmonar, ocasionado fraqueza muscular e restrição respiratória ${ }^{3,4}$.

Algumas patologias apresentam anormalidades que afetam a função respiratória muscular. A hipotonia e hipertonia, características das crianças portadoras de necessidades especiais, podem causar uma incapacidade de produzir força muscular respiratória, resultando em um padrão respiratório anormal ${ }^{5}$.
Cada portador de doenças, sendo criança ou não, necessita de um tratamento eficaz e específi$\mathrm{co}$, que trabalhe de forma completa as suas necessidades. Uma das formas de intervenção terapêutica é a equoterapia.

De acordo com a Associação Nacional de Equoterapia ANDE-Brasill ${ }^{6}$, equoterapia "[...] é um método terapêutico que utiliza o cavalo dentro de uma abordagem interdisciplinar nas áreas de Saúde, Educação e equitação, buscando o desenvolvimento biopsicossocial de pessoas com deficiência e/ou com necessidades especiais".

A utilização de atividades equestres como recurso terapêutico vem aumentando consideravelmente nas últimas décadas ${ }^{7}$. Apesar de ser uma prática antiga e muito utilizada, existe dificuldade de se encontrar um banco de dados com estudos científicos sobre o tema.

Dessa forma, há interesse em se realizar estudos que demostrem e quantifiquem a importância dos programas de equoterapia em crianças portadoras de necessidades especiais, o que pode interferir positivamente no quadro clínico e na qualidade de vida diária dos praticantes.

Em virtude das alterações e dos benefícios que a equoterapia traz para os praticantes, este estudo teve como objetivo verificar os efeitos da equoterapia sobre a capacidade respiratória em um grupo de crianças portadoras de necessidades especiais. 


\section{Materiais e métodos}

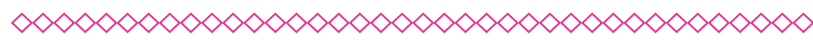

0 presente estudo caracterizou-se como do tipo longitudinal, de caráter intervencionista, e cunho qualiquantitativo. A população desta pesquisa foi composta por crianças com necessidades especiais, frequentadoras do Centro de Equoterapia Fortalecer - Patronato Agrícola Profissional São José, da região do Alto Uruguai Gaúcho, com uma amostra intencional de sete (7) participantes, de ambos os sexos, na faixa etária de 4 a 10 anos. Foram incluídas crianças que estavam na faixa etária estipulada; aquelas que apresentaram avaliação médica prévia autorizando à prática da equoterapia; que não praticavam equoterapia anteriormente e concordarem com o Termo de Consentimento Livre e Esclarecido (TCLE). Excluídas crianças que praticavam equoterapia anteriormente e não concordaram com o TCLE.

A avaliação inicial contou com o preenchimento de um questionário de identificação juntamente com o seu responsável, na qual consta, idade, sexo, déficit visual, cognitivo, auditivo, problemas respiratórios. Em seguida, foi mensurada a força muscular dos praticantes.

Para a mensuração da força muscular, foi utilizado o teste da manovacuometria, por meio do manovacuômetro digital modelo MVD 300, da marca Globalmed, portátil, a fim de identificar: (1) PIMáx a partir do volume residual (VR) e PEMáx a partir da capacidade pulmonar total (CPT).

As sessões de equoterapia foram iniciadas após a coleta dos dados. Os pacientes receberam atendimento uma vez por semana, em um período de dez semanas, com o tempo de 30 minutos cada sessão, totalizando dez sessões.

Ao início, foi realizado o envolvimento e a aproximação do praticante com o cavalo, orientando os cuidados ao tocar no animal, uso de gestos e palavras, e a recompensa ao animal durante e no final da sessão, por meio de agrados. Em seguida, foi realizado alongamento e relaxamento muscular, quando os praticantes relaxaram e sentiram o movimento rítmico do cavalo alongando coluna vertebral, membros inferiores e superiores. Realizado exercícios de amplitude de movimento e alinhamento postural, com bastão para adequação postural, boliche, bola no cesto, alcance da bola, argolas e atividades voltadas para o padrão respiratório do praticante.

Após esse período de dez sessões, os participantes foram reavaliados, conforme protocolo da avaliação inicial para análise dos resultados. Os dados foram analisados por estatística descritiva, em valores que demonstrarão escores, médias e percentuais de pré e pós-teste, devido ao pequeno número amostral. Foram tabulados em planilhas do Programa BioEstat.

Para a realização deste estudo, foram seguidas as diretrizes e normas regulamentadoras da Resolução 466/2012 do Conselho Nacional de Saúde do Ministério da Saúde, que trata de pesquisas envolvendo seres humanos. 0 estudo foi previamente submetido ao Comitê de Ética em Pesquisa da Universidade Regional Integrada do Alto Uruguai e das Missões - URI Erechim, e foi aprovado por meio do CAAE 47692215.9.0000.5351.

\section{Resultados}

$\infty \times \infty \times \infty \times \infty \times \infty \times \infty \times \infty \times \infty \times \infty \times \infty \times \infty$

Os participantes tiveram boa adesão ao programa de equoterapia durante as dez sessões oferecidas, com uma efetividade de $100 \%$ da participação. O estudo contou com portadores de: Síndrome de Asperger, Dispraxia Motora, Paralisia Cerebral Espástica Tetraplégica, Transtorno de déficit de atenção/hiperatividade.

Foram eliminadas duas crianças por não entenderem o comando (realizar as pressões respiratórias), sendo impossível a realização do teste; por isso, a amostra consistiu de 5 crianças.

A análise estatística foi realizada a partir do teste $t$ de Student para amostras pareadas, com nível de significância de $5 \%(p<0,05)$, no pacote estatístico BioEstat.

Em relação à força muscular, os resultados obtidos por meio da mensuração da manovacuometria, serão apresentados nas Figuras 1 e 2, representando respectivamente os pacientes em idade crescente - de quatro (4) a nove (9) anos.

Os valores de normalidade da PIMáx e PEMáx propostos por Domènech-Clar et al. ${ }^{8}$ para crianças, são considerados como PIMáx -68cmH2O e PEMáx 
$82 \mathrm{cmH} 20$ para sexo feminino e PIMáx $-79 \mathrm{cmH} 2 \mathrm{O}$ e PEMáx $95 \mathrm{cmH} 20$ para sexo masculino.

Neste estudo, foi demonstrado que, após a intervenção da equoterapia, houve melhoria da força muscular respiratória, tanto inspiratória quanto expiratória, mensurada respectivamente por meio da PIMáx e PEMáx, no teste de manovacuometria.

Como podemos visualizar nas Figuras $\mathbf{1}$ e $\mathbf{2}$, houve aumento da PIMáx e PEMáx em todos praticantes após a intervenção. Apenas um dos participantes diminuiu as pressões inspiratórias após a intervenção, de $-41 \mathrm{cmH} 20$ para $-40 \mathrm{cmH2O}$, não obtendo diferença significativa. Dois praticantes não realizaram o teste pelo motivo de não conseguirem entender o comando.

Através das amostras pareadas, verificou-se diferença significativa em PEMáx $(p=0,049)$. Nas PIMáx, não houve diferença significativa, mas constatou-se melhoria nas pressões respiratórias dos praticantes comparada ao início das intervenções.

\section{Discussão}

$\infty \times \infty \times \infty \times \infty \times \infty \times \infty \times \infty \times \infty \times \infty \times \infty \times \infty \times \infty$

Observou-se que, após as dez sessões de equoterapia, a força muscular dos praticantes meIhorou tanto para PEMáx como PIMáx; no entanto, mostrando-se com diferenças significativas em PEMáx.

Segundo Costa ${ }^{9}$, indivíduos com Síndrome de Down praticantes de equoterapia obtiveram meIhoria na força muscular respiratória, tanto inspiratória (PIMáx) como expiratória (PEMáx), quando comparados àqueles que não realizavam tratamento de equoterapia, embora para essas variáveis não tenha havido diferença significativa.

Santos, Lobo e Lourenço $^{10}$ afirmam que pacientes com Síndrome de Down apresentando hipotonia têm um fator associado ao déficit de força muscular do tronco, o que, por sua vez, resulta em perda da força muscular respiratória. Esses mesmos autores avaliaram a função respiratória em crianças com Síndrome de Down com idade entre 5 e 12 anos, revelando baixos valores de força muscular respiratória. Nosso estudo está de acordo com essas pesquisas e, além disso, foi verificada diferença significativa na variável PEMáx.
De acordo com Souza ${ }^{11}$, os valores de PIMáx e PEMáx dependem do volume pulmonar em que são realizadas as medidas e do correspondente valor da pressão de retração elástica do sistema respiratório, não apenas da força muscular respiratória. Assim, as mensurações dependem da compreensão das manobras realizadas e da vontade de cooperar e realizar movimentos e esforços respiratórios máximos. Dessa maneira, em nosso estudo, foram eliminadas duas crianças por não entenderem o comando de realizar as pressões respiratórias, o que as impossibilitou de realizarem os testes.

Hahn ${ }^{12}$ mensurou a capacidade respiratória de três indivíduos com síndrome de Down, submetidos a dez sessões de equoterapia antes e após a intervenção. Constatou-se um aumento na capacidade da força muscular respiratória de PEMáx e PIMáx, comprovando que a equoterapia contribuiu para a melhoria desta força. 0 movimento tridimensional do cavalo favorece a função respiratória, aumentando a capacidade pulmonar devido ao movimento.

Uma das manifestações do praticante é o ajuste tônico e, conforme os passos do cavalo, há uma contração e um relaxamento simultâneo dos músculos agonistas e antagonistas, permitindo uma melhor postura corporal, estímulo dos músculos respiratórios e consequente melhoria da capacidade pulmonar ${ }^{13,14}$. Dessa forma, os resultados desse estudo corroboram com os obtidos em nossa pesquisa.

\section{Considerações finais}

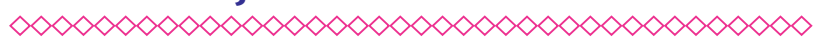

Os resultados obtidos permitem concluir que essa forma de tratamento pode contribuir para a capacidade respiratória dos praticantes, pois o movimento tridimensional do cavalo trabalha de forma sinergética e os resultados são alcançados de forma conjunta.

Os achados deste estudo reforçam a literatura, a qual sugere o uso da equoterapia como uma intervenção em potencial nas desordens da capacidade respiratória em portadores de necessidades especiais. 


\section{Referências}

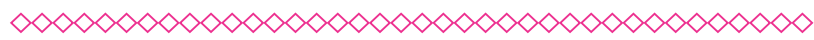

1. Costa D. Fisioterapia respiratória básica. São Paulo: Atheneu; 2004

2. Sarmento GJV (Org.). Fisioterapia respiratória no paciente crítico: rotinas clínicas. Barueri: Manole; 2005.

3. Azeredo CAC. Fisioterapia respiratória moderna. 4. ed. Barueri: Manole; 2002.

4. Severino FG, Resqueti VR, Bruno SS, Azevedo IG, Vieira RHG, Fregonezi GAF. Comparação entre o manovacuômetro nacional e o importado para medida da pressão inspiratória nasal. Revista Brasileira de Fisioterapia. 2010; 14(5):426-431.

5. Oliveira AS, Guerra RRS, Souza AT, Holanda RCAC, Soares FO. Estudo comparativo pneumofuncional entre crianças com síndrome de Down e não portadoras de síndrome. Revista Inspirar. 2004; 6(4):16-21.

6. Associação Nacional de Equoterapia. Curso básico de equoterapia. Brasília; 2004.

7. Lermontov T. A psicomotricidade na equoterapia. Aparecida: Ideias \& Letras; 2004.

8. Domènech-Clar R. et al. Maximal static respiratory pressures in children and adolescents. Pediatr. Pulmonol. 2003; 35(2):126-132.
9. Costa VSF. Influência da equoterapia na força muscular respiratória e coordenação motora global em indivíduos com Síndrome de Down no Distrito Federal. Dissertação (Mestrado em Educação Física) - Programa de Pós-Graduação Stricto-Sensu em Educação Física Universidade de Brasília, Faculdade de Educação física. Brasília; 2012.

10. Santos AM, Lobo VCT, Lourenço MGF. Perfil da Função Respiratória de Crianças Portadoras de Síndrome de Down na Faixa Etária de 5 a 12 anos de Idade. Fisioterapia Brasil. 2009; 10(3):153-158.

11. Souza RB. Pressões respiratórias estáticas máximas. Diretrizes para testes de função pulmonar. Jornal de Pneumologia: Publicação oficial da sociedade Brasileira de Pneumologia e Tisologia. 2002 out; 28(supl. 3).

12. Hahn L. Avaliação da capacidade funcional em pacientes com Síndrome de Down na Equoterapia. Novo Hamburgo, nov; 2011.

13. Martinez SL. Fisioterapia na equoterapia: análise de seus efeitos sobre o portador de necessidades especiais. São Paulo: Editora ideias e letras; 2005.

14. Pavei AZ. A equoterapia como recurso fisioterapêutico na doença de Parkinson. Trabalho de conclusão de curso (bacharelado em Fisioterapia). Universidade do Extremo Sul Catarinense. Criciúma; 2011. 


\section{Anexos}

Figura 1 - Valores de Pressão Inspiratória Máxima (PIMáx) obtidos através da manovacuometria, em dois momentos distintos, na avaliação e reavaliação da intervenção de equoterapia, representando respectivamente os pacientes em idade crescente.

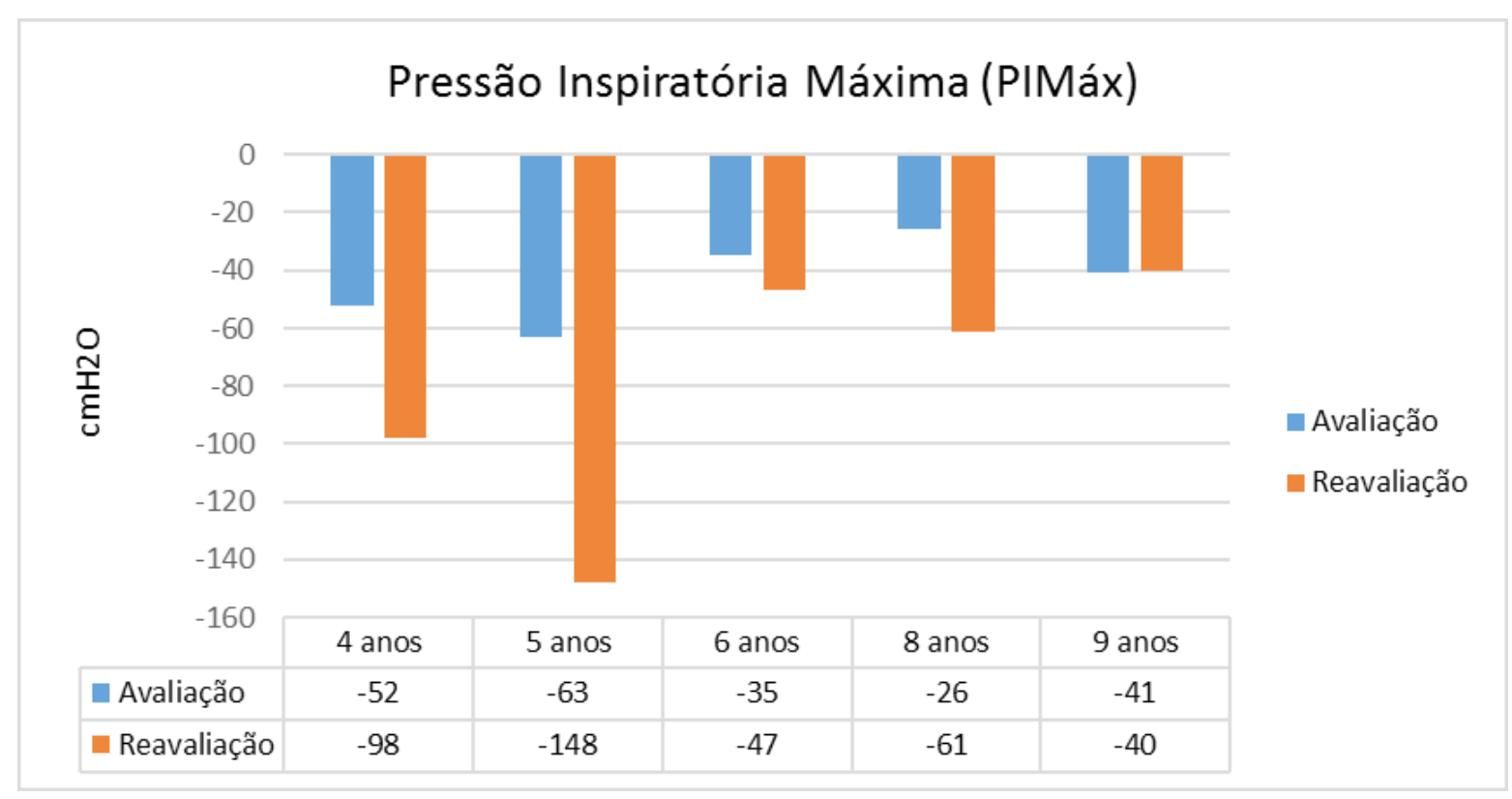

(clique para voltar ao texto)

Figura 2 - Valores de Pressão Expiratória Máxima (PEMáx) obtidos através da manovacuometria, em dois momentos distintos, na avaliação e reavaliação da intervenção de equoterapia, representando respectivamente os pacientes em idade crescente.

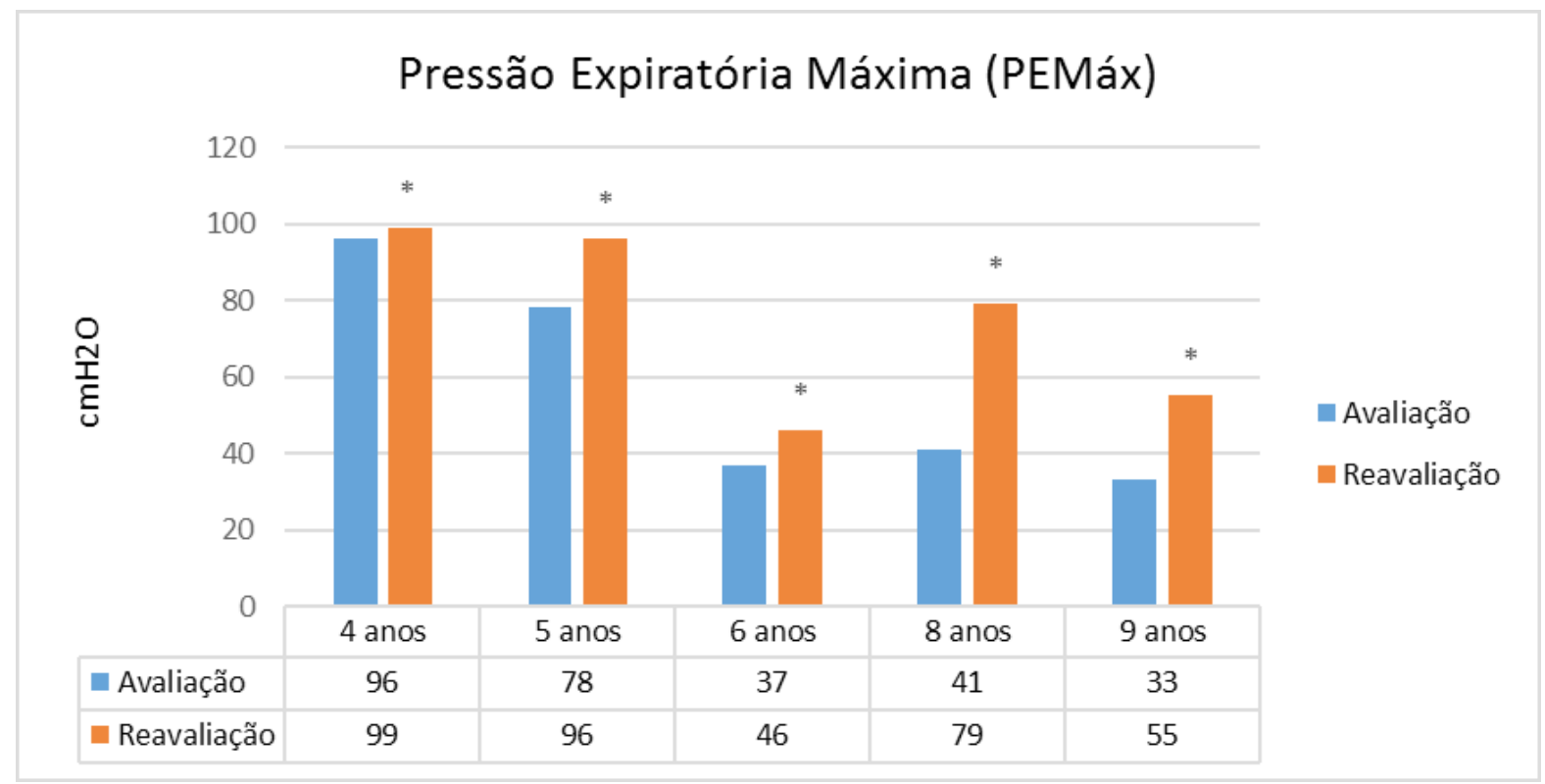

* Valor estatisticamente significativo $(p=0,049)$ 